Sluijs, E.M., Outinen, M., Wagner, C., Liukko, M., Bakker, D.H. de

The impact of legislative versus non-legislative quality policy in health care:

a comparison between two countries.

Health Policy: 58, 2001, nr. 2, p. 99-119

\begin{tabular}{|l|l|}
\hline Postprint Version & 1.0 \\
\hline Journal website & $\underline{\text { http://dx.doi.org }}$ \\
Pubmed link & $\begin{array}{l}\text { http://www.ncbi.nlm.nih.gov/entrez/query.fcgi?cmd=Retrieve\&db=pubmed\&dop } \\
\text { t=Abstract\&list_uids=11551661\&query_hl=53\&itool=pubmed_docsum }\end{array}$ \\
\hline DOI & $\begin{array}{l}\text { 10.1016/S0168-8510(01)00144-0 } \\
\text { This is a NIVEL certified Post Print, more info at http://www.nivel.eu }\end{array}$
\end{tabular}

\title{
The impact of legislative versus non-legislative quality policy in health care: a comparison between two countries
}

\author{
EMMY M. SLUIJS ${ }^{\mathrm{A}, *}$, MAARIT OUTINEN ${ }^{\mathrm{B}}$, CORDUlA WAGNER ${ }^{\mathrm{A}}$, MATTI LIUKKO ${ }^{\mathrm{C}}$, DinNY H. DE \\ BAKKER $^{\text {A }}$ \\ ${ }^{a}$ Nivel, The Netherlands institute for Health Services Research, PO Box 1568, 3500 BN, Utrecht, The \\ Netherlands \\ ${ }^{\mathrm{b}}$ National Research and Development Center for Welfare and Health STAKES, Helsinki, Finland \\ ${ }^{\mathrm{c}}$ Finnish Association of Local and Regional Authorities, Helsinki, Finland \\ * Corresponding author. Tel.: +31-30-2729700; fax: +31-30-2729729. E-mail address: e.sluijs@nivel.nl \\ (E.M. Sluijs).
}

\begin{abstract}
An important aim of the government's quality policy is to stimulate quality management (QM) in health care organizations. The relationship between the government's quality policy and QM in health care organizations is unknown. This article explores that relationship by comparing two countries with different quality policies, The Netherlands and Finland. In The Netherlands QM is required by law and health care is organized at national level. In Finland, QM is not required by law and the responsibilities for organizing health care are delegated to the municipalities. The question is whether or not these differences in national policy are reflected in the extent and effectiveness of QM in health care organizations in the two countries. A cross sectional survey was conducted in late 1999. Data about QM in both countries were gathered by questionnaire. The subsectors involved were hospitals, care for the disabled and care for the elderly. A total of 1172 health care organizations participated in the study (response rate 64\%). The results show that - in keeping with our hypothesis - slightly more QM-activities and more patient participation were found in Dutch health care organizations compared with the Finnish ones. However, contrary to our expectations, the Finnish organizations reported more perceived effects of their QM-activities. Further analyses showed that some QMactivities are more closely related to the effectiveness of QM than others. In particular, cyclic quality improvement procedures, human resource management and the flexible attitude of employees showed the strongest relationship with the perceived effects of QM. The difference between the national approach in The Netherlands and the decentralized approach in Finland did not, as we had assumed, result in more regional variation in QM in Finland. Conclusions: a government's quality policy may have some influence on the extent of QM in health care organizations. However, more QM-activities do not necessarily imply more effects. Recommendations: since QM-activities differ in the degree to which they bring about changes and improvements in care, it is recommended
\end{abstract}


Sluijs, E.M., Outinen, M., Wagner, C., Liukko, M., Bakker, D.H. de

The impact of legislative versus non-legislative quality policy in health care:

a comparison between two countries.

Health Policy: 58, 2001, nr. 2, p. 99-119

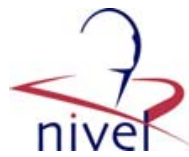

that policy makers promote those QM-activities, which are the most potent, in order to improve the quality of care.

\section{INTRODUCTION}

This article explores the impact of the government's quality policy on the extent and effectiveness of quality management (QM) in health care organizations.

In many countries, the national governments have expanded their traditional health policy by adding an explicit quality policy. This is in line with one of the World Health Organization's targets, that all countries should establish effective mechanisms for ensuring quality of patient care within their health care system. An important aim of the government's quality policy is to promote QM in health care organizations.

Both QM in health care organizations and quality policy developed by the governments are rather new phenomena in health care. As yet little is known about the impact of a quality policy on providers' behavior as far as QM is concerned. QM has been initiated and developed by health care providers $[1,2]$ and it is unknown whether or not it is also shaped by or related to the government's quality policy.

European countries differ in the quality policy they pursue [3]. This allows a comparison to be made between two European countries with different quality policies, the Netherlands and Finland. The main questions addressed are: are there differences between these countries in the extent and effectiveness of QM in health care organizations and are these differences related to the differences in quality policy of the governments?

Data about the extent and effectiveness of QM were collected in The Netherlands ( $n=671)$ and Finland $(n=501)$. In both countries, hospitals and institutions for the elderly and institutions for disabled were included.

\section{QUALITY MANAGEMENT (QM)}

In this article, QM is broadly defined as 'all the procedures explicitly designed to monitor, assess and improve the quality of care' (for example using guidelines, peer review, patient feedback, audits and so on). As yet, little is known about differences between and within countries with regard to QM in health care organizations. Although country reports exist [4-7], empirical results are scarce. Klazinga compared hospitals in 15 European countries and ascertained considerable differences between the countries in hospitals' QM activities [8]. Also, within a country, differences between health care organizations may exist. A national study in The Netherlands showed that the health care organizations differed in QM within each subsector of health care: for example, coherent quality systems were already operational in some hospitals or nursing homes, while in others such a system was still in its infancy or absent [9]. The implementation of QM appeared to be related to an innovative culture in the organization [10,11], a flexible attitude of employees and good collaboration between the management and professional practitioners in quality affairs [12,13]. It appears, therefore, that the implementation of QM is related to the cultural characteristics of a health care organization.

In general, high expectations exist about the effectiveness of QM in improving patient care [9]. The assumption is that well organized processes and systematic QM will reduce medical errors and adverse events and will result in appropriate and safe care (e.g. errorless patient safety).

Although many publications about QM exist, empirical effect-studies are scarce. The study of Shortell [10] among hospitals showed a positive relationship between the degree of QMimplementation and (perceived) outcomes. A review of the effectiveness of QM in nursing homes has also indicated improvements in some aspects in the process and outcomes of care [14]. However, in this review, only four controlled studies were found. The author concluded that at present it is unclear which QM-methods should be used in nursing homes to improve patient care [14]. Donabedian points to the imperfect state of our knowledge about the effects of the variety of quality assurance interventions [15]. He stated that "if we are truly committed to quality, almost any reasonable method will work'". In our study, we asked the management of the health care organizations about the perceived effects of QM in their institution. 
Sluijs, E.M., Outinen, M., Wagner, C., Liukko, M., Bakker, D.H. de

The impact of legislative versus non-legislative quality policy in health care:

a comparison between two countries.

Health Policy: 58, 2001, nr. 2, p. 99-119

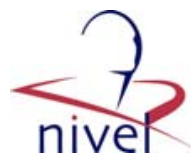

\section{COMPARABILITY OF THE TWO COUNTRIES}

In this study, a comparison between The Netherlands and Finland is considered to be justified because these countries are similar in many respects. Both are West European countries with well developed and comprehensive health care and social welfare systems [16]. In The Netherlands 8.6\% and in Finland 6.9\% of the Gross Domestic Product of both countries was allocated to health care in 1998 [17]. Life-expectancy is high in both countries: in The Netherlands men 75.2 and women 80.5; in Finland men 73.5 and women 80.8 in 1998 [17]. Health care is accessible for virtually the entire population through the national and private health insurance systems. In Finland, health care policy has emphasized the integration of health care and social welfare services since 1990.

Both countries share the problem of the increasing costs of health care. In recent years measures were taken to control these costs by cutting back (the budget for) the number of personnel and/or the number of hospital beds. Currently, an additional problem in The Netherlands is the shortage of nursing personnel in particular, and-connected with that-waiting lists for home care and nursing homes and for some hospital specialisms. There is not yet a serious shortage of personnel in Finland, but a shortage of doctors is emerging. Nevertheless, the providers in both countries were and are involved in a large number of quality projects.

\section{QUALITY POLICY IN THE NETHERLANDS AND FINLAND}

\subsection{Quality policy in The Netherlands}

In 1990, a new quality policy in The Netherlands was deemed necessary because of the government's plan to introduce self-regulation and competitive elements in the health care system. To protect patients in a system with less government regulation, the quality of care had to be ensured. At national level agreements, about a new quality policy were made among all parties involved: the providers, patient organizations, insurers and the government [18].

An important issue in the new policy was strengthening the position of patients and protecting their rights. To that end, three new Acts were implemented by the Ministry of Health, Welfare and Sports. A second major issue, addressed in two new Acts, is quality assurance by health care providers [19] ${ }^{1}$.

\subsubsection{Improving the position of the patient}

Basic patients' rights were adopted as part of The Netherlands Civil Code (Burgerlijk Wetboek) in 1995 in the Medical Treatment Agreements Act. These rights are firstly, the right to be informed about the illness, the possible treatments and their consequences and secondly, the right to give (or not to give) consent to proposed treatment. This right to 'informed consent' is closely linked to the right to information, because only well informed patients can choose between treatment options.

Also in 1995, the Clients' Right of Complaint Act came into effect. The Act requires care providers and care institutions to have an accessible complaints procedure in place. Currently, the great majority of providers do have a complaints committee with an independent chair, as the Act requires [20].

The third Act (Participation by Clients of Care Institutions Act, 1996) enables patients to influence the care institutions' policy. To this end, health care institutions are obliged to set up client councils. These client councils have considerable authority to make recommendations with regard to, for example, food, security, mergers and also with regard to the QM policy of the institution. In this way, client councils are supposed to contribute to improving the quality and client-centeredness of care. The result section of this article contains figures about the current state of affairs in practice.

\subsubsection{Quality requirements for professional practitioners and for institutions}

The quality of professional practitioners has been governed since 1998 by the Individual Health Care Professions Act. A statutory title protection and registration has been introduced for a number of professional practitioners, such as physicians, nurses, dentists and physical therapists. QM by practitioners is required. The Act also contains a number of provisions to protect patients against incompetent treatment. Various procedures, for example administering injections, are restricted to a limited group of professionals.

\footnotetext{
${ }^{1}$ www.minvws.nl (international health care quality of care documentation).
} 
Sluijs, E.M., Outinen, M., Wagner, C., Liukko, M., Bakker, D.H. de

The impact of legislative versus non-legislative quality policy in health care:

a comparison between two countries.

Health Policy: 58, 2001, nr. 2, p. 99-119

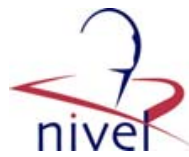

Quality requirements for health care organizations are laid down in the 1996 Care Institutions Quality Act. This Act requires all care organizations to set up a quality system to improve the quality of care. The quality system should reflect a cyclic process for monitoring, evaluating and (if necessary) improving the quality of care. The Act only gives a framework for QM but contains no norms for quality systems. It is up to institutions to develop their own quality system and choose their own QM procedures (for example using protocols and guidelines, peer review, audits, benchmarking, satisfaction surveys and so on).

The Quality Act, however, requires that all care institutions provide clarity on the quality of care by publishing an annual quality report (which must be sent to the Ministry, the Health Inspectorate and the regional patient/consumer organizations).

In summary, quality policy in The Netherlands is characterized by compulsory QM by providers and improved patient rights. (See result section for state of affairs).

\subsection{Quality policy in Finland}

Growing interest in the development of QM in Finland also began in the beginning of the 90s. At national level a quality policy was developed. This policy was based on 'steering by information'. No quality acts on QM for health care providers have been seen necessary. In stead of quality acts, four national recommendations on QM have been issued in the years 1994-1999. Earlier, an Act on patients right was passed in 1993.

\subsubsection{The position of patients and clients}

For the legal protection of consumers/patients, Finland was the first European country to enforce the Status and Rights of Patients Act in 1993. This Act has approximately the same focus and content as the Medical Treatment Agreements Act in The Netherlands, cited above. The Act defines a patient's right to good care and medical treatment and requires that every health care unit must appoint a patient ombudsman. A patient dissatisfied with the conduct of professionals providing health care can submit a complaint to the director responsible for the health care unit in question. If the complaint is not resolved within the organization, the patient can approach the State Provincial Offices and the National Authority for Medicolegal Affairs.

The Patient Injury Act safeguards patients' interests in the event of malpractice. All practitioners or their organizations must have patient insurance and patients are covered for bodily harm incurred in the course of health care or medical treatment (even if personnel cannot be shown to be legally responsible).

In contrast with the legislation in The Netherlands, none of these Finnish laws explicitly cites participation of patients or their organizations in quality management.

\subsubsection{National recommendations on quality management}

The Quality Council for Health Care (1993-1994) at STAKES published the first recommendation, Quality policy for health care in 1994 [21]. The second more detailed National Recommendation on QM in Social Welfare and Health Care [22] was prepared in collaboration with client, patient and professional organizations, service providers, sources of funding, and authorities and was published in late 1995.This recommendation was prepared on the basis of three main principles:

- QM has to be part of everyday work;

- a customer centered approach should be a key aspect of QM;

- information is a crucial tool for steering the implementation of QM.

The recommendation included a quality vocabulary for health care and social welfare, based on ISO 8402 vocabulary aimed at creating a more homogeneous use of the concepts and terms.

The third and fourth recommendations were published in 1998 and 1999 respectively. The third concerned the Recommendation on QM of health services provided and purchased by municipalities [23], drafted by the Association of the Finnish Association of Local and Regional Authorities. The most recent recommendation on QM in Social Welfare and Health Care for the 21st Century [24] was published in April 1999. The detailed recommendations are organized according to eight topics:

1. customer participation in QM;

2. leadership for the steering of quality; 
3. personnel as a prerequisite for high quality;

4. QM for preventive as well other activities;

5. management of processes as a basis for QM;

6. information as a basis for the continuous enhancement of quality;

7. systematization of QM;

8. support for individual action recommendation and quality criteria.

In addition to the recommendations, there are several quality related acts, defining aspects of quality or quality management, for example, the Act on Private Health Care Providers or the Act on the Professional Health Care Personnel. The latter Act was revised in 1994. It aims at furthering the protection of patients and the quality of health care services by ensuring an educated work force and the registration and monitoring of the personnel. Title protection has been introduced for a wider amount of personnel than anywhere else in Europe, 38 professions. The Act is closely linked to the Act on the Status and Rights of Patients.

\subsubsection{Decentralized approach}

Another difference vis a`vis The Netherlands concerns health care policy in Finland, which is characterized by a decentralized organization of health care services and a diminishing role for the central government. The State is a part financier and gives only general recommendations and instructions concerning the way in which the services are provided. Decisions are taken at regional and local level and the responsibility for organizing the health care and social welfare services is delegated to the municipalities.

In summary, the government's quality policy in Finland can be characterized as steering by issuing national recommendations on QM.

\subsection{Hypotheses}

This study is based on three hypotheses, which are derived from the main differences in quality policy of the two countries.

The first difference concerns the degree of obligatory regulations imposed on health care providers. In The Netherlands, 'quality' laws have been enacted, imposing a number of QM procedures on care providers. No quality legislation has been passed in Finland, but national recommendations on QM have been issued. The quality policy in Finland is based on voluntary cooperation of health care providers. From this difference, we have hypothesized that compared with Finland, more QM procedures are carried out in Dutch organizations, because it is required by law. As a consequence we expect that Dutch organizations also perceive more effects of QM compared with the Finnish ones.

A second difference concerns participation of patients in QM. In both countries, the position of patients has been strengthened in recent years. In The Netherlands, additional legislative measures were taken to promote the participation of patients and patient organizations in health care. Hence, the hypothesis to be tested is that patient participation is further developed in The Netherlands.

The third difference concerns the centralized approach in The Netherlands versus a decentralized approach in Finland. In The Netherlands, quality policy is pursued at national level, which means that government regulations are the same for all health care providers in the country. In Finland, regional variation in health care may exist. The responsibilities for the organization and quality of care are delegated to local and regional authorities. The municipalities are entitled to pursue their own policy and adjust it to regional circumstances. The third hypothesis, therefore, is that more regional variation in QM exists in Finland compared with The Netherlands.

This article has been structured along these hypotheses and contains three result sections. The first section describes the differences between the countries in QM activities and the perceived effectiveness of QM. In the second section, explanations are sought for the differences in effectiveness. This section shows which QM procedures are most strongly related to the effectiveness of QM. Finally, the third section concerns the regional variation of QM. 
Sluijs, E.M., Outinen, M., Wagner, C., Liukko, M., Bakker, D.H. de

The impact of legislative versus non-legislative quality policy in health care:

a comparison between two countries.

Health Policy: 58, 2001, nr. 2, p. 99-119

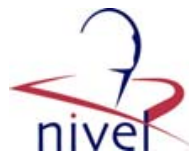

\section{METHODS}

The study was conducted in close collaboration between three parties: Nivel (Netherlands institute for Health Services Research), STAKES in Finland (National Research and Development Center for Welfare and Health) and the Finnish Association of Local and Regional Authorities.

Descriptions of the national quality policies were based on documentation and on expert knowledge of the researchers. The state of affairs regarding QM in both countries was based on an identical questionnaire. A total of 1872 questionnaires were sent (in the autumn of 1999) to all the hospitals, all the institutions for disabled people and to (a random sample of) institutions for the elderly in The Netherlands and Finland. All hospitals were approached, including university hospitals, central hospitals and regional hospitals. Institutions for disabled included inpatient and ambulant care for physically and mentally disabled people. Institutions for the elderly are nursing homes and service homes for the elderly. The addressee was the management.

An existing validated questionnaire was used, developed in TheNetherlands in 1995 and improved in $1999[12,13]$. The questionnaire had a closed, Likert-type format with two to four ordinal scaled options per item. The reliability and validity of the questionnaire were described elsewhere [12]. For Finland, the questionnaire was translated into the Finnish language. Finnish health care experts checked the comprehensibility of the concepts used.

The questionnaire contained five subjects: QM-activities, patient participation, positive and negative side effects of QM, characteristics of the organization and hospital region of the organization.

\subsection{QM-activities}

In the questionnaire, 38 items asked about QM-activities. These items were empirically clustered into four scales, with the reliability coefficients given below, namely:

- the availability of quality policy documents (six items; Alpha.79);

- human resource management (11 items; Alpha.72);

- using protocols and guidelines (seven items; Alpha.64);

- quality improvement procedures (14 items; Alpha.82).

The specific items are given in Table 2. For the items in the first three scales, we use the confirmative answers (yes, 'it' is present). In the fourth scale, the items had three options as follows, the procedure (1) is not present; (2) is present but not cyclic; (3) present and cyclic. The definition of cyclic (e.g. systematic) was - the results are systematically used to make improvements (for example the information obtained from peer review, audits, satisfaction surveys). Only the third option 'cyclic' was used in this article. Thus, this scale reflects the cyclic character of QM.

\section{[ TABLE 2 ]}

\subsection{Patient participation}

Five items concern the degree to which patients or patient organizations were involved in the quality management of the institution (Alpha.72). For example: are patients or patient organizations involved in the development of quality criteria or guidelines? Are they participating in quality projects? Are they involved in evaluating the results of the institution's quality policy and so on (see Table 3 for the specific items). In The Netherlands, a question was added about the presence of a client council (required by law). This question was left out in the Finnish version because client councils are rare and not required in Finland.

\section{[ TABLE 3 ]}

\subsection{Positive and negative effects of $Q M$}

On the one hand, we asked if QM already had resulted in positive effects (12 items; Alpha.87).

Examples of those effects are - improvements in processes and outcome of care, improved patient centeredness, a better image of the institution, improved satisfaction of patients and personnel, cost reduction (see Table 4 for specific items). 
Sluijs, E.M., Outinen, M., Wagner, C., Liukko, M., Bakker, D.H. de

The impact of legislative versus non-legislative quality policy in health care:

a comparison between two countries.

Health Policy: 58, 2001, nr. 2, p. 99-119

\section{[ TABLE 4 ]}

On the other hand, there were four items asking about increased workload, rising costs or disheartened employees due to side-effects of QM. These four items could not be scaled and are dealt with separately (see Table 5).

\section{[ TABLE 5 ]}

\subsection{Characteristics of the organization}

To describe the characteristics of the organization, firstly the size of the organization was asked for in full time equivalents. Secondly, some questions were added about factors, which are supposed to contribute to the effectiveness of QM (for example flexible attitude of employees). And some questions about possible hindering circumstances (for example, excessive workload; high level of absenteeism).

These items were scored on a 5-point scale, ranging from 'not applicable' to 'very applicable'. (See Table 7 for specific items).

\section{[ TABLE 7 ]}

\subsection{Regional division of health care}

To investigate regional differences in QM the institutions were grouped into the hospital regions (24 in Finland and 27 in The Netherlands). In Finland, the regional number was filled in by the institutions. In The Netherlands the postal codes (ZIP-code) were used to assign institution per region. To provide for a mix of institutes in each region, we only included the regions with more than 15 health care institutions in the analyses (19 regions in The Netherlands and 16 in Finland).

\subsubsection{Analyses}

The data of both countries were processed in a common database at Nivel. To test differences between The Netherlands and Finland, chi square tests were used for nominal data and t-tests for data at ratio level. The reliability of the scales was tested with Cronbach's Alpha (figures reported above). The correlations between the scales are expressed in Pearson's correlation coefficients. The relationship between QM-activities (independent variable) and the perceived effects of QM (dependent variable) were investigated by using stepwise regression analysis. Finally, multi level analysis was used to test the assumption that more regional variation in QM-activities exists in Finland, compared with The Netherlands. MLN-analysis adjusts the data for differences in the number of cases (per region).

All missing values were transformed into zero, assuming that missing implied that the activity was 'not present' in the institution. The level of significance is $P<0.01$ throughout the article.

\subsubsection{Respondents}

A total of 1172 questionnaires were returned. The overall response is $64 \%$. The response rate in The Netherlands is $68 \%$ and in Finland 59\%. The highest response was obtained from hospitals in Finland (71\%) and the lowest from the institutions for the elderly in Finland (56\%).

In The Netherlands, $55 \%$ of the questionnaires were completed by the management and $44 \%$ by the quality coordinator (1\% 'others'). In Finland, these percentages are 53 and 12\%, respectively, (35\% by 'others') (Table 1). 
Sluijs, E.M., Outinen, M., Wagner, C., Liukko, M., Bakker, D.H. de

The impact of legislative versus non-legislative quality policy in health care:

a comparison between two countries.

Health Policy: 58, 2001, nr. 2, p. 99-119

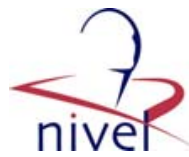

\section{[ TABLE 1 ]}

\section{RESULTS}

\subsection{The extent and effectiveness of quality management}

\subsubsection{Quality management in The Netherlands and Finland}

Table 2 gives an overview of $38 \mathrm{QM}$-activities listed in the questionnaire and shows the percentage of institutions in The Netherlands and Finland performing these activities (Table 2).

6.1.1.1. Quality policy documents. Significantly more institutions in The Netherlands report that they have drawn up quality policy documents such as mission statements, a quality policy for the institution and quality action plans. The majority of the institutions in The Netherlands publish an annual quality report (83\%), which is required by law. In Finland, 24\% publish such a report.

6.1.1.2. Human resource management. Minor differences between the countries exist with regard to human resource management. There are seven significant differences in QM-activities, three in favor of The Netherlands and four in favor of Finland. Particularly in Finland, more institutions report that staffs are encouraged to increase their expertise that new staff is selected with a positive attitude to $\mathrm{QM}$ and that staff (and new staff) is trained in QM.

6.1.1.3. Protocols and guidelines. Significantly more protocols and guidelines are reported in The Netherlands compared with Finland. For example protocols for (parts of) medical treatment, for medical aids or critical incidents. In Finland, more institutions have protocols for patient information.

6.1.1.4. Cyclic quality improvement procedures. It must be noted that the figures in the fourth scale only represent those institutions, which report that the results of the QI-procedures are systematically used to make improvements. In general, the table shows that few institutions in both countries report such a cyclic mechanism in quality management and the differences between the countries are rather small. It appears that in Finland, more institutions are using staff satisfaction surveys to make improvements, whilst in The Netherlands, the complaints registration is more frequently used for that purpose.

Comparing the total sum of QM-activities, the t-test showed a mean of 15.8 (S.D. 5.8) QM-activities in Dutch institutions and a mean of 13.3 (S.D. 7.3) in the Finnish ones $(P<0.01)$.

It can be concluded that the hypothesis that more institutions in The Netherlands, report QMactivities compared with Finland cannot be rejected. Some nuances deserve attention. In The Netherlands, more emphasis is put on quality documents and guidelines, whilst in Finland more emphasis is placed on human resource management. Cyclic quality management is still in its infancy in both countries.

\subsubsection{Patient participation}

The second hypothesis is that patient participation is further developed in The Netherlands because this was a major issue in the government's policy. Table 3 shows the comparison between The Netherlands and Finland (Table 3).

Significantly more institutions in The Netherlands compared with Finland report that patients or patient organizations are involved in quality management activities. For example, in nearly half the institutions (49\%) patient (organizations) participates in the development of quality criteria. In $44 \%$ of the institutions, patient (organizations) is involved in evaluating the degree to which the institutions' quality targets are met. These figures are better understood, knowing that in The Netherlands, it is often the client council, which participates in quality management. A client council has been established in $77 \%$ of the institutions (not in the table) [13]. In Finland, client councils are rare and not obliged.

We conclude that the second hypothesis has been confirmed. More patient participation is seen in The Netherlands compared with Finland. 
Sluijs, E.M., Outinen, M., Wagner, C., Liukko, M., Bakker, D.H. de

The impact of legislative versus non-legislative quality policy in health care:

a comparison between two countries.

Health Policy: 58, 2001, nr. 2, p. 99-119

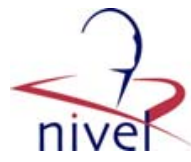

\subsubsection{Effectiveness of quality management}

Effectiveness of QM is expressed in positive effects and negative side effects. In the questionnaire 12 possible positive effects of QM were listed. The respondents could mark which effect (if any) had been reached as a result of QM. Table 4 shows the results for The Netherlands and Finland (Table 4).

Significantly more institutions in Finland report that QM has resulted in positive effects compared with The Netherlands. Improvement of care processes is reported by $26 \%$ of the institutions in The Netherlands and 29\% in Finland (no significant difference between the two countries). The most frequently cited effects of quality management are increased client centeredness and client satisfaction (both cited by 38\% of the institutions in Finland). Cost savings as a result of QM are seldom reported in either country (cited by $3-14 \%$ of the institutions). Relatively large differences between The Netherlands and Finland exist with regard to increased staff satisfaction and staff dedication: these effects are cited twice as often in Finland compared with The Netherlands (Table 4).

Significantly fewer negative side effects of QM are reported by Finnish institutions compared with those in The Netherlands (Table 5). QM appears to increase the workload in $43 \%$ of the Dutch institutions and in 23\% of the Finnish institutions. This may possibly be partly due to the increased regulations and guidelines in Dutch organizations. Three quarters (74\%) of Dutch institutions (not in the table) report an expanded number of regulations whereas in Finland this is reported by $37 \%$. As a second negative side effect, (the development of) QM requires extra money, especially in The Netherlands (reported by $28 \%$ of the institutions).

The hypothesis that more QM-activities imply more positive effects in The Netherlands must be rejected.

\subsection{Factors related to the effectiveness of quality management}

\subsubsection{Differences in effectiveness of QM explored}

Summarizing the results, so far the following may be concluded with regard to the differences between the countries.

In The Netherlands, QM is characterized by an emphasis on documentation, regulation and client participation while at the same time fewer positive effects are reported compared with Finland.

In Finland, QM is characterized by fewer quality activities and an emphasis on human resource management while at the same time more positive effects are reported compared with The Netherlands.

To investigate which factors are most strongly related to the effectiveness of QM regression analyses have been carried out. In this analyses, we are using the sum scores of the scales. The intercorrelations between the scales are given in Table 6, separately for The Netherlands and Finland (Table 6).

\section{[ TABLE 6 ]}

All correlations between the QM-scales are significant at the $P<0.01$ level. The relations are rather weak in The Netherlands and stronger in Finland. This implies that in Finland more than in The Netherlands coherence exists in the QM activities within an organization. Coherence means that, for example attention paid to quality documents also implies that attention is paid to the other three QMactivities [9,13]. Of the QM-activities, cyclic quality improvement procedures show the strongest relation with the perceived effects in both countries ( 0.43 in The Netherlands and 0.54 in Finland). Stepwise multiple regression analyses were carried out to investigate the contribution of the QMscales to the perceived effects. The dependent variable is the sum of effects. The main independent variables are the above-cited four QM-scales and client participation.

Besides, we added the following factors, which could have a positive or negative influence on the effectiveness of QM.

Organizational characteristics:

- the size of the institution (in full time equivalents);

- the degree of competition experienced.

Cultural characteristics: 
Sluijs, E.M., Outinen, M., Wagner, C., Liukko, M., Bakker, D.H. de

The impact of legislative versus non-legislative quality policy in health care:

a comparison between two countries.

Health Policy: 58, 2001, nr. 2, p. 99-119

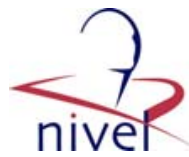

- flexible or conservative attitude of employees;

- centralized or decentralized decision making;

Impeding circumstances:

- excessive workload;

- long waiting lists;

- vacancies that cannot be filled;

- high level of absenteeism.

- organizational turmoil after merger or change.

All these items were scored on a 5-point scale (see method section) (Table 7).

Table 7 shows that $28 \%$ of the variance can be explained by six factors in The Netherlands. In Finland, 39\% of the variance can be explained by five factors. In both countries the cyclic quality improvement procedures are most potent in explaining the perceived effects of QM. Besides, in both countries human resource management and the flexible attitude of employees make a substantial contribution to the effectiveness of QM. The positive influence of the flexible attitude of employees was found in other studies $[9,10]$.

Only in Finland is client participation weakly but significantly related to the effectiveness of QM. In The Netherlands, the negative relationship with an excessive workload and absenteeism cannot be denied.

The cyclic QI-procedures deserve further analysis to investigate the contribution of each of the 11 QI-procedures to the effectiveness. A second regression analysis was carried out with effects as dependent variable and the 11 cyclic QI-procedures separately as independent variables.

The results (not in a table) show that a significant contribution to the effectiveness in both countries could be attributed to three cyclic QI-procedures - (1) patient satisfaction surveys; (2) needs analysis of clients; (3) internal audits. These three variables explain $12 \%$ of the effectiveness in The Netherlands and 21\% in Finland.

\subsection{Regional variation in quality management}

The third and last hypothesis is that greater regional variation in QM exists in Finland compared with The Netherlands. The sum of QM-activities was used as the dependent variable. A Multi Level Analysis was carried out because the QM-activities are at the institutions level (level 1) while an explanation is sought at the regional level (level 2). A stepwise approach was followed.

In the first step, we tested for whether regional differences existed at all. The results showed small but significant differences between regions ( $5 \%$ of the variance was attributed to regional differences; $P<0.01)$. The second step tested for whether the regional differences remained, after the differences between the countries were taken into account. The results showed that regional differences in The Netherlands were absent and small regional differences remained in Finland. However, these differences no longer reached the level of significance (regression coefficient 0.47 , standard error 0.45 , Chi square $\left.\left(\chi^{2}\right) 1.04\right)$.

The conclusion is that the third hypothesis - more regional differences in Finland — must be rejected.

\section{DISCUSSION}

This study has shown that detailed comparisons between countries can be made with regard to QM in health care organizations, despite the differences in health care systems and health policy. Moreover, these differences provide for opportunities to gain more insight into the implications of different quality policies. As yet, little is known about those implications, because an explicit governmental quality policy is a relatively recent phenomenon in health care.

Comparisons between the government's quality policy and its consequences may be of particular relevance in the European countries. Health ministers strive at co-operation [3] as a consequence of article 39 of the EU treaty, which permits free movement of people among the member states including patients and health workers. According to Belcher [25], the European Union has an increasing influence on the way health care is delivered at national level and some endeavors are already directed at harmonizing quality requirements and 'best practices' among the member states. 
Sluijs, E.M., Outinen, M., Wagner, C., Liukko, M., Bakker, D.H. de

The impact of legislative versus non-legislative quality policy in health care:

a comparison between two countries.

Health Policy: 58, 2001, nr. 2, p. 99-119

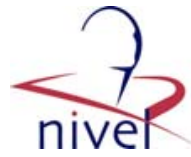

This study shows that similarities and differences in quality management between countries can be traced.

Before we turn to the implications of our findings, the strengths and weaknesses of the study are discussed. The strength of the study is the measurement instrument, which was identical for both countries and thoroughly tested and refined in former research $[9,12,13]$. The instrument provides for detailed information about QM, independent of the quality model used by an organization (ISO, BSC, EFQM or other models). It is applicable to institutions of different subsectors in health care, owing to the fact that many items reflect general mechanisms of (good) operational management in an organization. Nevertheless, improvements to the instrument should be sought in two directions. Firstly, the development of quantifiable indicators is recommended, to gain more insight into the factual quality and safety of patient care and thus the effectiveness of QM. Secondly, the instrument could be perfected if the core of quality management could be measured. We are aware that the sum of QI-activities does not necessarily reflect the core of (total) quality management in an organization.

However, we do not know whether total quality management can be measured by questionnaire at all.

A weakness of the study is the self reported data, which is inherent in surveys conducted by questionnaire. Social desirability in some answers cannot be ruled out. The results of a non-response analysis (conducted by telephone) in The Netherlands showed that the non-respondents reported fewer QM-activities compared with the respondents. Therefore, a slightly positive bias in the results cannot be excluded.

A second weakness concerns the respondents' positions in their organization. The questionnaire was sent to the management of the institutions because they are responsible for QM and have an overview of QM. Whether their opinions are shared by the professional practitioners and patients is unknown. This could be resolved by a multiple-source approach, but that would be a complex and very timeconsuming enterprise, especially when the survey covers several countries. The approach followed in this study may suffice to give an impression of QM at the national level.

The main question addressed in this study concerns the impact of legislative versus non-legislative quality policy. We assumed that more QI-activities occurred and that patients were more involved in QM in The Netherlands because of the legislative quality policy in The Netherlands and the nonlegislative approach in Finland. This hypothesis was confirmed. The greatest differences between the two countries were seen in those QM-activities, which are required by law in The Netherlands: publication of an annual quality report, the client councils and the involvement of patients in QM and complaints registration. From these findings, we conclude that legislation has specific effects. The overall effects of legislation are unknown. This cross sectional study does not allow for cause and effect conclusions.

There is not a big difference between the two countries in the total amount of QM-activities and some QM-activities occurred more in Finland, for example concerning human resource management. The Finnish national recommendations on QM may possibly have had the same stimulating influence as the legislative framework on QM in The Netherlands. We assume that the development of QM is partly driven by health care practitioners and providers themselves, because a great number of quality projects are seen in both countries. As yet, the exact impact of the national quality policy on the development of QM is unclear and is an interesting subject for further research.

In this study, the respondents were asked which effects were reached as a consequence of QM. In general, it appeared that the positive effects were particularly related to specific QM-activities: namely to 'cyclic quality improvement procedures'. This is congruent with general quality theories, which emphasize the importance of systematic and continuous improvements based on information about results. In both countries, the positive effects could also be attributed to the attention paid to human resource management and to a flexible attitude of employees. From this, we conclude that the quality of patient care depends significantly on the care for and attitude of the employees.

There are differences between the two countries in the reported effectiveness of QM. Although more QI-activities were seen in The Netherlands, more positive and fewer negative side-effects were reported in Finland. At first sight, the results were puzzling. Further analyses yielded three possible explanations. Firstly, the finding that Finnish institutions pay considerable attention to human resource management may partly account for the reported positive effects of QM in Finland. In The Netherlands, many institutions are faced with personnel shortages and long waiting lists, resulting in a 
Sluijs, E.M., Outinen, M., Wagner, C., Liukko, M., Bakker, D.H. de

The impact of legislative versus non-legislative quality policy in health care:

a comparison between two countries.

Health Policy: 58, 2001, nr. 2, p. 99-119

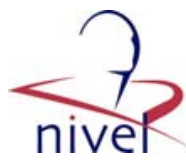

(too) heavy workload, which factors were found to be weakly but negatively related to the effects of

QM. In Finland, the workload of health care staff is also heavy and there is a shortage of personal.

Although the reasons for that may vary between The Netherlands and Finland. In Finland, there is unemployment in health care (for example nurses), but the municipalities or hospitals don't seem to employ staff enough. Maybe the main reason for that is the lack of money.

Secondly, it appeared that more coherence in QM activities existed in the Finnish organizations compared with the Dutch ones. The degree of coherence of a quality system may partly account for the effectiveness of QM. Some indications for this assumption were found in the Dutch studies [9,13]. A small number of institutions with a coherent and well developed quality system reported over twice as many improvements in care processes as well as in the outcomes of care, compared with the majority of the institutions in which the quality system was still in the process of development. The third explanation is more speculative. In The Netherlands it was found that in the last five years substantial progress was made with regard to the development of quality documents, protocols and guidelines [13]. This may account for the reported negative side-effects ('bureaucracy') in The Netherlands. As yet it is unknown whether or not bureaucratic activities reflect a necessary step in the development of a quality system, which many Finnish institutions have yet to undergo. After all, the processes to manage patient care and safety must be clear, before systematic quality control can take place.

Despite our imperfect knowledge about the developmental phases of quality management, a recommendation to avoid superfluous bureaucracy seems justified.

Health policy in the two countries differs in the way responsibilities for health care are assigned - in Finland this responsibility is delegated to regional authorities (municipalities), whereas The Netherlands follows a national approach. From this difference, we hypothesized that in Finland more regional variation in QM should occur, compared with The Netherlands. This hypothesis was not confirmed by the results. Regional differences were absent in The Netherlands and very small in Finland. Interestingly, a recent Finnish study shows that the variations between patient groups and areas in relation to the amount of services provided have narrowed since the decentralization of the responsibilities in 1993 [26]. These are important findings in respect of ongoing debate in both countries about the pros and cons of a national versus regional organization of health care. In Finland, there are discussions about a more centralized approach. In The Netherlands, there is a tendency towards a more decentralized approach, with the delegation of some responsibilities to regional health insurers. In the knowledge that many governments nowadays are seeking a balance between steering and deregulating, it may be helpful to know that a decentralized approach - at least as described in this article - does not necessarily result in substantial variations in quality management within health care organizations.

\section{ACKNOWLEDGEMENTS}

This project was co-funded by The Netherlands Organization for Scientific Research, Council for Medical and Health Research (MW-NWO) and The Academy of Finland, Research Council for Health.

\section{TABLES}

Table 1

Number and percentages of respondents for each subsector in The Netherlands $(N=671)$ and Finland $(N=501)$

\begin{tabular}{|c|c|c|c|c|c|c|c|c|c|}
\hline \multirow[t]{2}{*}{ Sectors } & \multicolumn{3}{|c|}{ The Netherlands } & \multicolumn{3}{|c|}{ Finland } & \multicolumn{3}{|l|}{ Total } \\
\hline & Sent & Response & $\%$ & Sent & Response & $\%$ & Sent & Response & $\%$ \\
\hline Hospitals & 149 & 101 & 68 & 83 & 59 & 71 & 232 & 160 & 69 \\
\hline Care of the disabled & 267 & 182 & 69 & 122 & 84 & 69 & 389 & 266 & 68 \\
\hline Care of the elderly & 568 & 388 & 68 & 638 & 358 & 56 & 1206 & 746 & 62 \\
\hline Total & 984 & 671 & 68 & 843 & 501 & 59 & 1827 & 1172 & 64 \\
\hline
\end{tabular}


Sluijs, E.M., Outinen, M., Wagner, C., Liukko, M., Bakker, D.H. de The impact of legislative versus non-legislative quality policy in health care: a comparison between two countries.

Health Policy: 58, 2001, nr. 2, p. 99-119

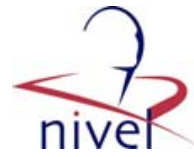

Table 3

Percentage of institutions with patients or patient organizations participating in quality management in The Netherlands $(N=671)$ and in Finland $(N=501)$

The Netherlands (\%)

Finland $(\%)$

Patient (organizations) participation

Evaluation of achievement of quality targets

In development of criteria

$44 \quad 21 *$

In quality committees

$49-10^{*}$

In quality improvement projects

$41-8 *$

In development in protocols or guidelines

$3810^{*}$

$* P<0.01$.

Table 4

Percentage of institutions reporting positive effects achieved as a result of QM in The Netherlands $(N=671)$ and Finland $(N=501)$

\begin{tabular}{lllll}
\hline Positive effects achieved & The Netherlands (\%) & $n^{\mathrm{b}}$ & Finland (\%) & $n$ \\
\hline Clients & & & & \\
Increased client centeredness & 25 & 653 & $38^{\mathrm{a}}$ & 410 \\
Increased client satisfaction & 18 & 651 & $38^{\mathrm{a}}$ & 440 \\
Staff & & & 440 \\
Increased staff satisfaction & 11 & 649 & $30^{\mathrm{a}}$ & 440 \\
Increased dedication of employees & 16 & 633 & $31^{\mathrm{a}}$ & 436 \\
Organization & & & & 424 \\
Improved manageability of organization & 15 & 644 & $24^{\mathrm{a}}$ & 404 \\
Improved image of organization & 17 & 650 & $23^{\mathrm{a}}$ & \\
Improved satisfaction of external parties & 13 & 634 & $25^{\mathrm{a}}$ & 441 \\
Care & & & & 426 \\
Improved quality of care processes & 26 & 656 & 29 & \\
Improved quality of outcomes/results & 15 & 648 & $22^{\mathrm{a}}$ \\
Productivity & & & & 428 \\
Improved productivity & 11 & 628 & $18^{\mathrm{a}}$ & $14^{\mathrm{a}}$ \\
Diminished costs in own organization & 6 & 625 & $13^{\mathrm{a}}$ & 393 \\
Diminished costs elsewhere & 3 & 533 \\
\hline
\end{tabular}

a Significance $P<0.01$.

$\mathrm{b}$ The number of respondents differ per item. 
Sluijs, E.M., Outinen, M., Wagner, C., Liukko, M., Bakker, D.H. de

The impact of legislative versus non-legislative quality policy in health care:

a comparison between two countries.

Health Policy: 58, 2001, nr. 2, p. 99-119

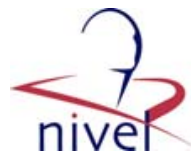

Table 2

Percentage of institutions with QM-activities in The Netherlands $(N=671)$ and in Finland $(N=501)$

Quality management activities

The Netherlands (\%) Finland $(\%)$

Quality policy documents

Mission statement

$47^{\mathrm{a}}$

Product description

Quality policy

Quality action plan for the organization

Annual quality report

Quality manual

Human resource management

Staff is encouraged to increase expertise $\quad 80 \quad 91^{\text {a }}$

Time allocated for quality activities $\quad 78 \quad 64^{\mathrm{a}}$

Feedback to staff about results $\quad 23 \quad 28$

New staff selected on positive attitude $\quad 35 \quad 45^{\mathrm{a}}$

Management trained in quality management $\quad 70 \quad 66$

$\begin{array}{lll}\text { Staff trained in quality management } & 74 & 87^{\mathrm{a}}\end{array}$

$\begin{array}{lll}\text { Training based on quality policy } & 41 & 22^{\mathrm{a}}\end{array}$

New staff trained in quality management $\quad 9 \quad 20^{\mathrm{a}}$

Management explains quality requirements $\quad 60 \quad 55$

Management monitors quality procedures $\quad 37 \quad 34$

Management controls compliance with quality procedures $\quad 41 \quad 22^{\mathrm{a}}$

Protocols and guidelines

For parts of medical treatment $\quad 90 \quad 73^{\mathrm{a}}$

For patient information $\quad 47 \quad 59^{\mathrm{a}}$

For medical aids $\quad 66 \quad 54^{\mathrm{a}}$

For critical incidents (in care process) $\quad 48 \quad 38^{\mathrm{a}}$

$\begin{array}{lll}\text { For diagnostic related groups } & 41 & 37\end{array}$

For the routing of the client $\quad 50 \quad 40^{\mathrm{a}}$

$\begin{array}{lll}\text { For cooperation with other providers } & 43 & 44\end{array}$

Cyclic quality improvement procedures ${ }^{\mathrm{b}}$

Monodisciplinary peer review

$\begin{array}{lll}\text { Multidisciplinary peer review } & 10 & 12\end{array}$

Care plan management $\quad 61 \quad 51^{\mathrm{a}}$

Job assessment interviews $\quad 57 \quad 52$

$\begin{array}{lll}\text { Internal audits } & 10 & 10\end{array}$

Inter-institutional visits $\quad 7 \quad 5^{\text {a }}$

Management information systems $\quad 12 \quad 10^{\mathrm{a}}$

$\begin{array}{lrr}\text { User satisfaction surveys } & 29 & 34\end{array}$

Referrer satisfaction surveys $\quad 3 \quad 8^{\text {a }}$

$\begin{array}{lll}\text { Staff satisfaction surveys } & 17 & 35^{\mathrm{a}}\end{array}$

Needs surveys of potential users $\quad 5 \quad 11^{\text {a }}$

Referrers need surveys $\quad 4 \quad 6$

Complaints registrations $\quad 43 \quad 22^{\text {a }}$

Benchmarking

${ }^{\text {a }}$ Significant $P<0.01$.

${ }^{\mathrm{b}}$ Cyclic, results of QI-procedures are systematically used to make improvements. 
Sluijs, E.M., Outinen, M., Wagner, C., Liukko, M., Bakker, D.H. de The impact of legislative versus non-legislative quality policy in health care: a comparison between two countries.

Health Policy: 58, 2001, nr. 2, p. 99-119

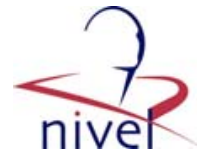

Table 5

Percentage of institutions reporting negative side effects of QM in The Netherlands $(N=671)$ and in Finland $(N=501)$

\begin{tabular}{lcc}
\hline & The Netherlands $(\%)$ & Finland (\%) \\
\hline Increased workload & 43 & $23^{\mathrm{a}}$ \\
Increased costs & 28 & $5^{\mathrm{a}}$ \\
Diminished flexibility employees & 7 & $2^{\mathrm{a}}$ \\
Diminished motivation/satisfaction of staff & 3 & 1 \\
No negative side effects & 36 & $49^{\mathrm{a}}$ \\
\hline
\end{tabular}

a Significance $P<0.01$.

Table 7

Results of the stepwise multiple regression analysis with positive effects as dependent variable in The Netherlands $(N=671)$ and in Finland $(N=501)$

\begin{tabular}{lccc}
\hline Independent variables $^{\mathrm{a}}$ & $\mathrm{B}$ & Beta & $R^{2}$ \\
\hline The Netherlands & & & 0.23 \\
Cyclic QI procedures & 0.28 & 0.23 & 0.21 \\
Human res. management & 0.25 & -0.14 & 0.24 \\
Excessive workload & -0.37 & 0.16 & 0.27 \\
Decentralized decisions & 0.39 & 0.11 & 0.28 \\
Flexible attitude of employees & 0.28 & -0.09 & 0.29 \\
Excessive absenteeism & -0.18 & & 0.29 \\
Adjusted $R^{2}=0.28$ & & & 0.34 \\
Finland & & 0.28 & 0.37 \\
Cyclic QI procedures & 0.36 & 0.21 & 0.39 \\
Quality documents & 0.40 & 0.15 & 0.40 \\
Flexible attitude of employees & 0.53 & 0.14 & 0.10 \\
Human res. management & 0.19 & & \\
Client participation & 0.31 & & \\
Adjusted $R^{2}=0.39$ & & & \\
\end{tabular}

a Significant $P<0.01$. 
Sluijs, E.M., Outinen, M., Wagner, C., Liukko, M., Bakker, D.H. de

The impact of legislative versus non-legislative quality policy in health care:

a comparison between two countries.

Health Policy: 58, 2001, nr. 2, p. 99-119

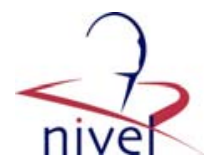

Table 6

Pearson correlations between the scales concerning QM, client participation and effects, in The Netherlands $(N=671)$ and Finland $(N=501)$

\begin{tabular}{|c|c|c|c|c|c|c|c|}
\hline & & $\begin{array}{l}\text { Quality } \\
\text { documents }^{\mathrm{a}}\end{array}$ & $\begin{array}{l}\text { Human res. } \\
\text { management }^{\mathrm{a}}\end{array}$ & $\begin{array}{l}\text { Protocols/guidelines } \\
\text { a }\end{array}$ & $\begin{array}{l}\text { Cyclic Q1 } \\
\text { procedures }^{\mathrm{a}}\end{array}$ & $\begin{array}{l}\text { Client } \\
\text { participation }^{\mathrm{a}}\end{array}$ & Positive effects \\
\hline \multirow[t]{2}{*}{$\begin{array}{l}\text { H.r. } \\
\text { management }{ }^{b}\end{array}$} & Ned & 0.31 & - & & & & \\
\hline & Fin & 0.51 & & & & & \\
\hline \multirow{2}{*}{$\begin{array}{l}\text { Protocols/ } \\
\text { guidelines }\end{array}$} & Ned & 0.28 & 0.33 & - & & & \\
\hline & Fin & 0.45 & 0.47 & & & & \\
\hline \multirow{2}{*}{$\begin{array}{l}\text { Cyclic Q1 } \\
\text { procedures }\end{array}$} & Ned & 0.38 & 0.40 & 0.28 & - & & \\
\hline & Fin & 0.59 & 0.53 & 0.35 & & & \\
\hline \multirow{2}{*}{$\begin{array}{l}\text { Client } \\
\text { participation }\end{array}$} & Ned & 0.19 & 0.30 & 0.15 & 0.28 & - & \\
\hline & Fin & 0.31 & 0.36 & 0.30 & 0.27 & & \\
\hline \multirow[t]{2}{*}{ Positive effects } & Ned & 0.23 & 0.37 & 0.22 & 0.43 & 0.17 & - \\
\hline & Fin & 0.50 & 0.46 & 0.35 & 0.54 & 0.30 & \\
\hline
\end{tabular}

a Significant $P<0.01$.

${ }^{\mathrm{b}}$ Human resource management. 
Sluijs, E.M., Outinen, M., Wagner, C., Liukko, M., Bakker, D.H. de

The impact of legislative versus non-legislative quality policy in health care:

a comparison between two countries.

Health Policy: 58, 2001, nr. 2, p. 99-119

\section{REFERENCES}

1. Collopy BT. Do Doctors Need Deming? Quality Assurance in Health Care 1993;5:3-5.

2. Harvey G. Quality in health care: traditions, influences and future directions. International Journal for Quality in Health Care 1996;8:341-50.

3. Quality in Health Care. Opportunities and Limits of Co-operation at EU-level Aimed at Achieving a High Standard of Health Protection in the Member States. Conference Report of European Health Ministers, Austria, 1998.

4. Shaw CD. Quality assurance in the United Kingdom. Quality Assurance in Health Care 1993;5:107-18.

5. Garpenby P. Implementing quality programmes in three Swedish county councils: the views of politicians, managers and doctors. Health Policy 1997;39:195-206.

6. Schilling J, Kapetanios E, Mayrhofer M, Faisst K, Norrie M, Haudenschild C. The Swiss database on quality projects in medical institutions. Health Policy 2000;51:101-7.

7. Ovretveit J. A comparison of approaches to health service quality in the UK, USA and Sweden and of the use of organizational audit frameworks. European Journal of Public Health 1994;4:46.

8. Klazinga N. Concerted Action Programme on Quality Assurance in Hospitals -1993 (COMAC/ HSR/QA). Global Results of the Evaluation. International Journal for Quality in Health Care 1994;6:219-30.

9. Casparie AF, Sluijs EM, Wagner C, de Bakker DH. Quality systems in Dutch health care institutions. Health Policy 1997;42:255-67.

10. Shortell SM, O'Brien JL, Carman JM, Foster RW, Hughes EFX, Boerstler H, O'Connor EJ. Assessing the impact of continuous quality improvement/total quality management: concept versus implementation. Health Services Research 1995;30:377-401.

11. Wagner C. Implementation and effectiveness of quality systems in Dutch health care organizations (Thesis). Utrecht, Nivel, 1999.

12. Wagner $\mathrm{C}$, de Bakker $\mathrm{DH}$, Groenewegen PP. A measuring instrument for evaluation of quality systems. International Journal for Quality in Health Care 1999;11:119-30.

13. Sluijs EM, Wagner C. Quality systems in health care organizations. The state of affairs in 2000 (in Dutch). Utrecht, Nivel, 2000.

14. Wagner C, Wal G van der, Groenewegen PP, Bakker DH de. The effectiveness of elements of quality systems in nursing homes: a review (in press).

15. Donabedian A. The effectiveness of Quality Assurance. International Journal for Quality in Health Care 1996;8:401-7.

16. Vienonen MA. Health and Health Care System in The Netherlands. An international Comparison. In: Schrijvers AJP (ed). Health and Health Care in The Netherlands. Utrecht, De Tijdstroom, 1997:20-32.

17. OECD Health Data 2000.

18. Casparie AF. View from The Netherlands. Quality in Health Care 1993;2:138-41.

19. Ministry of Health, Welfare and Sports in the Netherlands: www.minvws.nl(internationalhealthcare- quality of care-documentation).

20. Friele RD, Ruiter $\mathrm{C}$ de, Wijmen $\mathrm{F}$ van, Legemaate J. Evaluation of the Clients' Right of Complaint Act (in Dutch). Den Haag, Zorg Onderzoek Nederland (ZON), 1999.

21. Quality policy for health care in Finland. Quality Council for Health Care. Helsinki, STAKES, 1994.

22. QM in social welfare and health care. National Recommendation on QM in Social Welfare and Health Care. Helsinki, STAKES, 1995.

23. Recommendation on QM of health services provided and purchased by municipalities. Association of Finnish Local and Regional Authorities. Helsinki, 1998.

24. QM in Social Welfare and Health Care for the 21st Century. National Recommendation, Ministry of Social Affairs and Health, STAKES, Association of Finnish Local and Regional Authorities, Helsinki 1999.

25. Belcher PJ. The role of the European Union in Health care: overview. Zoetermeer, Council for Health and Social Service (RVZ), 1999.

26. Hannu Uusitalo $\mathrm{CH}$, Parpo A, Hakkarainen A (eds). Social Welfare and Health Care Service Review 2000. National Research and Development Center for Welfare and Health. Reports 250, Helsinki, 2000. 